\title{
Influência da Concentração de Inóculo e da Idade da Planta na Intensidade de Doença Causada por Fusarium graminearum em Egeria densa e E. najas*
}

\author{
Carlos R. Borges Neto ${ }^{1}$, Cláudia Q. Gorgati ${ }^{2}$ \& Robinson A. Pitelli ${ }^{1}$ \\ ${ }^{1}$ Departamento de Biologia Aplicada à Agropecuária, ${ }^{2}$ Departamento de Engenharia Rural, Faculdade de Ciências \\ Agrárias e Veterinárias de Jaboticabal, Universidade Estadual Paulista, Via de Acesso Prof. Paulo Donato \\ Castellane, s/nº, CEP 14884-900, Jaboticabal, SP
}

(Aceito para publicação em 12/03/2004)

Autor para correspondência: Carlos R. Borges Neto

BORGES NETO, C.R., GORGATI, C.Q. \& PITELLI, R.A. Influência da concentração de inóculo e da idade da planta na intensidade de doença causada por Fusarium graminearum em Egeria densa e E. najas. Fitopatologia Brasileira 29:282-288. 2004.

\section{RESUMO}

Um isolado de Fusarium graminearum estudado na UNESP/ Campus de Jaboticabal, onde foi comprovada sua eficácia no controle de Egeria densa e E. najas, macrófitas aquáticas submersas. Para estudar o efeito de diferentes concentrações do inóculo na severidade de doença, foram conduzidos experimentos em incubadoras para BOD, com concentrações variando em um décimo, de 0,1 até 1,4 g/l de arroz moído colonizado com $F$. graminearum. Para verificar os possíveis efeitos da idade da planta sobre a severidade de doença, plantas com, no mínimo $35 \mathrm{~cm}$, de comprimento foram excisadas em segmentos correspondentes às idades de crescimento. Os tratamentos com concentrações de inóculo a partir de $0,5 \mathrm{~g} / \mathrm{l}$ apresentaram sintomas. Todos os tratamentos inoculados com o fungo, nas concentrações a partir de $0,5 \mathrm{~g} / \mathrm{l}$, apresentaram drástica redução na produção de biomassa fresca. Todos os segmentos utilizados como plantas-teste ( 0 a $32 \mathrm{~cm}$ de comprimento) apresentaram suscetibilidade ao fungo. Os ponteiros de plantas de ambas as espécies apresentaram maior severidade de sintomas no sexto e oitavo dias após a inoculação, contudo, os segmentos correspondentes às idades 2 e 3 apresentaram maior redução de biomassa fresca quando inoculadas, apesar de não apresentarem sintomas tão severos quanto a idade 1 . Com relação ao ganho de biomassa fresca, as testemunhas apresentaram sempre maior crescimento do que os respectivos tratamentos inoculados, contudo os segmentos correspondentes à idade 4 apresentaram menor ganho de biomassa fresca do que as demais idades.

Palavras-chave adicionais: biocontrole, plantas aquáticas, fatores bióticos.

\section{ABSTRACT}

Effects of inoculum concentrations and plant age in Fusarium graminearum disease development on Egeria densa and $E$. najas

A very promising isolate of Fusarium graminearum is being studied in the UNESP/Campus de Jaboticabal, where it was proven to be effective in the control of Egeria densa and E. najas, the submerged aquatic macrophytes. To study the effect of different concentrations of inoculum in disease severity, experiments have been conducted in Biochemical Oxigen Demand (BOD) incubators with concentrations varying from one-tenth, of 0.1 up to $1.4 \mathrm{~g} / 1$ in rice colonized with $F$. graminearum mycelia. To verify the possible effect of the age of the plant on disease severity, plants with at least $35 \mathrm{~cm}$ of length, were excised in diverse segments corresponding to the age of growth. The treatments with concentrations of inoculum from
$0.5 \mathrm{~g} / \mathrm{l}$ had presented symptoms. All the treatments inoculated with fungi, in concentrations from $0.5 \mathrm{~g} / \mathrm{l}$, presented drastic reduction in the production of fresh biomass. All the segments used as planttest (0-32 cm length) presented susceptibility to fungus. The top of the leaves of both the species at age 1 had the most severe disease symptoms at the sixth and eighth days after the corresponding inoculation. However, segments to ages 2 and age 3 presented the greatest reduction of fresh biomass at day eight, although symptoms were not severe as in age 1 . With relation to the profit of fresh biomass, the control had always presented bigger growth over the respective inoculated treatments, however the corresponding segments to age 4 had presented minor profit of fresh biomass than the other ages.

\section{INTRODUÇÃO}

O processo de eutrofização dos corpos hídricos pelo enriquecimento das águas com esgotos urbanos, industriais e da agricultura, provoca alterações físico-químicas que promovem e sustentam a produtividade da vegetação aquática, criam

\footnotetext{
*Parte da tese de doutorado do primeiro autor. Faculdade de Ciências Agrárias e Veterinárias de Jaboticabal/UNESP (2003). Parcialmente financiada pela CAPES.
}

condições para a deterioração da qualidade da água e iniciam uma sucessão na qual algumas macrófitas são favorecidas em detrimento de outras (Correl \& Correl, 1975; Anderson, 1998).

Outros fatores que contribuem para o profuso crescimento de macrófitas aquáticas são: (i) redução das pressões bióticas negativas exercidas pelos inimigos naturais e competidores, (ii) assoreamento de rios, lagos e reservatórios e (iii) alteração do regime de fluxo da água (Pitelli, 1998; Pitelli et al., 2000). 
Influência da concentração de inóculo e da idade da planta...

No Brasil, duas espécies Egeria densa Planch. e Egeria najas Planch., membros da família da Hydrocharitaceae (Lorenzi, 2000), têm-se tornado problemáticas em reservatórios de usinas hidrelétricas, sendo responsáveis por queda na geração de energia e aumento do custo da mesma devido à necessidade de constantes operações de controle (Tanaka, 1998).

Um isolado de Fusarium graminearum Schwabe temse mostrado patogênico às espécies acima citadas e de suficiente especificidade para que se avalie a possibilidade de seu desenvolvimento como agente de controle biológico. Este fungo vem sendo estudado no Laboratório de Controle Biológico de Plantas Daninhas "Prof. Dr. Giorgio de Marines", da Faculdade de Ciências Agrárias e Veterinárias - FCAV, da Universidade Estadual Paulista - UNESP, como agente potencial de controle biológico pela estratégia inundativa (Nachtigal, 2000; Mendes 2002).

O controle biológico de plantas daninhas é uma técnica que utiliza organismos vivos para controlar ou reduzir populações de espécies de plantas indesejáveis (Van Den Bosh et al., 1987). A estratégia do bioherbicida envolve o aumento da efetividade do organismo candidato, por meio de aplicações inundativas de esporos ou outros propágulos de um determinado patógeno em concentrações ideais, a fim de gerar um alto nível de doença, com conseqüente morte ou supressão de crescimento da planta daninha em questão.

Estudos sobre as interações patógeno x hospedeiro, observando e identificando os eventos pré e pós-infecção, são essenciais para o melhor entendimento dos processos ligados à patogênese de $F$. graminearum. Estes estudos aliados a observações sobre o desenvolvimento da doença em diferentes condições de ambiente, são de importância fundamental para o estabelecimento de técnicas e procedimentos que tornem as condições epidemiológicas favoráveis ao patógeno e, consequientemente, ao controle da planta daninha (Charudattan \& Walker, 1982; Te Beest, 1991).

Estudos têm sido conduzidos para várias interações patógeno $\mathrm{x}$ hospedeiro tanto em ambientes controlados como em condições de campo, na tentativa de determinar as condições ótimas sob as quais os organismos seriam mais efetivos no controle biológico (Te Beest \& Templeton, 1985). Assim, a idade da planta hospedeira na qual ela se torna mais suscetível, bem como a concentração de inóculo adequada para o estabelecimento de altos níveis de doença, devem ser definidas para cada associação patógeno x hospedeiro.

Pouco se conhece sobre a patologia em plantas aquáticas e não há expressivo conhecimento de microflora associada às espécies em questão. $\mathrm{O}$ biocontrole de $E$. densa está sendo investigado em algumas partes do mundo, utilizando-se peixes, moluscos e fungos (Anderson, 1998), sendo considerado efetivo, com adequações ao tipo de habitat, além do baixo custo e do reduzido impacto ambiental. No Brasil, levantamentos sistemáticos de inimigos naturais de ambas as espécies foram efetuados em 1995, em regiões do Pantanal Mato-grossense e nos Rios Paraná e Tietê, pela equipe de controle biológico de plantas daninhas da Faculdade de
Ciências Agrárias e Veterinárias da UNESP (Nachtigal, 2000). Com base em observações do referido autor, efetuadas no curso desses levantamentos, a estratégia inundativa de controle biológico foi considerada a mais adequada, uma vez que existiam poucas evidências para ocorrências de epidemias em condições naturais.

Dentre os organismos selecionados, destacou-se um isolado de $F$. graminearum considerado altamente promissor, por proporcionar elevados danos às duas macrófitas, com suficiente seletividade (Nachtigal, 2000). Este fungo é oriundo da Micoteca da Universidade Federal Rural de Pernambuco UFRPE e vem sendo intensivamente estudado como agente de controle biológico pela estratégia inundativa (Nachtigal, 2000; Mendes, 2002).

O presente trabalho faz parte desta pesquisa global e visa estudar as influências das condições bióticas, como concentração de inóculo e idade da planta, na intensidade de doença causada pelo fungo sobre as duas macrófitas. Estes conhecimentos são de fundamental importância para se estabelecer as condições ótimas de infecção e do real potencial do agente de biocontrole.

\section{MATERIAL E MÉTODOS}

O isolado de $F$. graminearum utilizado foi cedido pelo Laboratório de Fitopatologia da Universidade Federal Rural de Pernambuco - UFRPE. Quando de sua entrada no Banco de Germoplasma de Fungos Fitopatogênicos do Laboratório de Controle Biológico da FCAV - UNESP, recebeu o código FCAV\#940. As colônias foram mantidas em tubos de ensaio contendo o meio BDA, sob refrigeração $\left(4{ }^{\circ} \mathrm{C}\right)$. Foram efetuadas transferências de colônias para placa de Petri contendo o mesmo meio. A patogenicidade do isolado foi assegurada por meio de inoculações periódicas nas plantas hospedeiras e recuperação de colônias em meio de cultura.

Para obtenção do inóculo, o fungo foi cultivado em arroz, conforme descrito por Nachtigal (2000): $200 \mathrm{~g}$ de arrozparboilizado, com grãos longos (Uncle Ben’s ${ }^{\circledR}$ ), misturados com $120 \mathrm{ml}$ de água destilada em frascos Erlemmeyers de $1.000 \mathrm{ml}$ de capacidade. Este conjunto foi autoclavado por 20 min a $120{ }^{\circ} \mathrm{C}$ e pressão de 1 atm. Após a autoclavagem e resfriamento, três discos de micélio-ágar, retirados das margens de colônias cultivadas por 14 dias, a $25^{\circ} \mathrm{C}$ e fotoperíodo de 12 h diárias de luz, foram transferidos para cada frasco.

Após 14 dias de incubação em estufa para Biochemical Oxygen Demand (BOD), a $25^{\circ} \mathrm{C}$ e fotoperíodo de $12 \mathrm{~h}$ diárias de luz, procedeu-se a transferência do conteúdo dos frascos para bandejas ( $32 \mathrm{~cm}$ de comprimento x $23 \mathrm{~cm}$ de largura $\mathrm{x} 4$ cm de altura) revestidas com papel alumínio. As bandejas permaneceram por sete dias dentro de câmara apropriada (fotoperíodo de $12 \mathrm{~h}$ diárias de luz, $25 \pm 2{ }^{\circ} \mathrm{C}$, com umidade do ar variando de 50 a $65 \%$ ) com circulação forçada de ar para secagem.

Após a secagem, os substratos colonizados com micélio foram moídos em moinho de facas (Marconi, MA 340) e as partículas resultantes, constituídas de micélio e substrato, 
C.R. Borges Neto et al.

foram separadas quanto a granulometria, utilizando-se apenas as menores que $0,5 \mathrm{~mm}$. Tais partículas, com densidade de 10 $\mathrm{g} / \mathrm{cm}^{3}$, foram armazenadas a $4^{\circ} \mathrm{C}$, em refrigerador.

Foram coletados plantas das duas espécies, E. densa e E. najas, no Reservatório da Usina Hidrelétrica de Três Irmãos, localizada no rio Tietê (20³9'S; 51 $18^{\circ}$ 'W), e Souza Dias, localizada no rio Paraná, em Jupiá - SP (24º $48^{\prime}$ S; $\left.51^{\circ} 18^{\prime} \mathrm{W}\right)$, para utilização nos experimentos. Foram realizadas coletas periódicas de propágulos de acordo com a necessidade de plantas. Utilizaram-se ponteiros medindo $8 \mathrm{~cm}$ de comprimento das plantas coletadas.

Os ponteiros de cada espécie tiveram a biomassa fresca determinada, individualmente, após remoção do excesso de água da superfície das folhas por contato em papel absorvente. Os ponteiros foram transferidos para tubos de ensaio com 2,5 $\mathrm{cm}$ de diâmetro e $20 \mathrm{~cm}$ de comprimento. As plantas foram cultivadas em solução nutritiva de Clark modificada, com 1/5 da força iônica (Clark, 1975). Para a inoculação, $0,7 \mathrm{~g}$ de arroz moído colonizado com micélio de $F$. graminearum foi suspensa em um litro de solução de Clark. Alíquotas de $50 \mathrm{ml}$ desta suspensão foram colocadas em tubos de ensaio onde as plantas foram submersas e tiveram contato com o potencial agente de controle biológico em suspensão. Nas testemunhas, os procedimentos adotados foram os mesmos, mas foram utilizados apenas grãos de arroz moídos, sem colonização pelo fungo. Após a inoculação as plantas foram acondicionadas em incubadoras para BOD.

\section{Concentração de Inóculo}

Para o estudo do efeito de diferentes concentrações do inóculo (arroz moído colonizado com micélio) na severidade de doença foram utilizados tratamentos com concentrações variando em um décimo, de 0,1 até 1,4 g/l. Foram incluídos, como testemunhas, as mesmas quantidades utilizando-se apenas arroz moído. Os ensaios com diferentes concentrações de inóculo foram conduzidos apenas com a espécie $E$. densa, pois na época de sua realização, plantas-teste de $E$. najas não foram encontradas nas coletas em campo.

\section{Idade da Planta}

Devido a grande dificuldade em se determinar a idade destas espécies de macrófitas uma vez que sua propagação se dá vegetativamente por pequenos fragmentos do caule (segmentos com apenas $0,5 \mathrm{~mm}$ podem originar novas plantas), partiu-se da premissa que os segmentos encontrados em profundidades diferentes seriam cronologicamente mais antigos dos que os encontrados próximos à superfície da água. Deste raciocínio e para verificar os possíveis efeitos da idade da planta sobre a severidade de doença, foi utilizada a seguinte metodologia: plantas, com no mínimo $35 \mathrm{~cm}$ de comprimento, foram excisadas em diversos segmentos, com $8 \mathrm{~cm}$ de comprimento, correspondentes às idades de crescimento. Para efeitos de avaliação foi considerada: Idade 1 - ponteiros de 8 $\mathrm{cm}$ de ambas as espécies, que corresponderiam aos tecidos mais jovens; Idade 2 - segmentos coletados de $8 \mathrm{~cm}$ a $16 \mathrm{~cm}$ de profundidade; Idade 3 - segmentos coletados de $16 \mathrm{~cm}$ a
$24 \mathrm{~cm}$ de profundidade e idade 4 - segmentos coletados de 24 cm a $32 \mathrm{~cm}$ de profundidade. Utilizou-se inóculo na concentração de $0,7 \mathrm{~g} / \mathrm{l}$ e foram incluídas plantas-testemunhas de cada idade, cultivadas em solução nutritiva adicionada de arroz moído, na mesma concentração do inóculo.

As avaliações dos sintomas foram realizadas a cada dois dias, até o oitavo dia após a inoculação, com o auxílio de escala de notas (Tabela 1) modificada de Nachtigal (2000). A partir das notas foram calculados os Índices de Infecção, que transformam os dados adimensionais em porcentagem de área foliar com sintomas, utilizando a fórmula proposta por Mckinney (1923):

Índice de infecção $=\frac{\Sigma(\text { grau de escala x freqüência }) \times 100}{\text { (Número total de unidades x grau máximo da escala) }}$

A variação da biomassa fresca da planta, antes e depois da inoculação, também foi avaliada.

Para todos os ensaios, o delineamento experimental adotado foi o de blocos ao acaso com três repetições. Todos os ensaios foram repetidos três vezes no tempo, constituindo os blocos do delineamento. A cada montagem dos ensaios, cada tratamento teve cinco tubos de ensaio e foi utilizada a média das notas e da porcentagem de biomassa fresca obtidas nas avaliações para constituir o dado de cada parcela experimental. Para as análises estatísticas, os dados de porcentagem de ganho de biomassa fresca foram transformados em arc-seno $\sqrt{\mathrm{x} / 100}$. Os dados foram submetidos à análise não linear e as curvas de progresso da doença foram obtidas utilizando-se a equação de Boltzmann:

$$
Y=\frac{\mathrm{A}_{1}-\mathrm{A}_{2}}{1+e^{\left(\mathrm{x}-\mathrm{x}_{0}\right) / \mathrm{dx}}}+A_{2}
$$

Onde $\mathrm{A} 1$ = ponto de mínimo da curva, $\mathrm{A} 2$ = ponto de máximo da curva, $x_{0}=$ centro, $d x=$ constante de tempo. As equações das curvas de progresso da doença foram obtidas utilizando-se o programa Origin $®$ 6.0.

\section{RESULTADOS E DISCUSSÃO}

\section{Concentração de Inóculo}

O inóculo na concentração de $0,1 \mathrm{~g} / \mathrm{l}$ não foi suficiente para iniciar o processo infetivo (Tabela 2). Aos dois dias após a inoculação, apenas os tratamentos com concentrações maiores ou iguais a $0,7 \mathrm{~g} / \mathrm{l}$ apresentaram sintomas da doença, no quarto dia, os tratamentos com concentrações a partir de $0,5 \mathrm{~g} / \mathrm{l}$ apresentaram sintomas, contudo, no intervalo de $0,5 \mathrm{a}$ $0,9 \mathrm{~g} / \mathrm{l}$ não houve diferença estatisticamente significativa, o intervalo de 1,1 a 1,3 g/l foi superior ao intervalo anterior, com exceção do tratamento com concentração de $1,4 \mathrm{~g} / \mathrm{l}$. No sexto dia após a inoculação os tratamentos 0,3 e $0,4 \mathrm{~g} / \mathrm{l}$ que não haviam mostrado sintomas na avaliação anterior apresentaram 2,22\% de área foliar com sintomas (Tabela 2). Os demais tratamentos seguiram a mesma tendência da avaliação anterior, contudo com maior progresso da doença, 
Influência da concentração de inóculo e da idade da planta...

TABELA 1 - Escala de notas adotadas nas avaliações de sintomas provocados por Fusarium graminearum, isolado FCAV\#940, em plantas de Egeria densa e E. najas, modificada de Nachtigal (2000)

\begin{tabular}{ll}
\hline \hline Nota & Descrição \\
\hline 0 & Sem sintomas \\
1 & Clorose aparente não superior a 5\% do tamanho da brotação \\
2 & $\begin{array}{l}\text { Amarelecimento foliar visível na porção basal ou,eventual- } \\
\text { mente, na porção superior, não afetando mais de 20\% do } \\
\text { tamanho da brotação }\end{array}$ \\
3 & $\begin{array}{l}\text { Amarelecimento afetando os tecidos a té a porção mediana } \\
\text { ou terço superior das brotações }\end{array}$ \\
4 & $\begin{array}{l}\text { Amarelecimento do caule e de mais de } 80 \% \text { da área foliar; } \\
\text { flacidez dos tecidos vegetais e, eventualmente, abscisão dos } \\
\text { segmentos terminais }\end{array}$ \\
& $\begin{array}{l}\text { Amarelecimento generalizado com completa desintegração } \\
\text { dos tecidos vegetais }\end{array}$ \\
\hline
\end{tabular}

chegando ao máximo de 44,44\% de área foliar com sintomas com inóculo na concentração de 1,4 g/l. No oitavo dia após a inoculação os tratamentos incluídos no intervalo de 1,0 a 1,3 g/l apesar de apresentarem diferentes porcentagens de área foliar com sintomas, mas não foram estatisticamente diferentes, destacando-se apenas o tratamento com concentração igual a 1,4 g/l (Tabela 2). As equações das curvas de progresso de doença são similares para os diversos tratamentos onde foi possível realizar a análise não linear (Tabela 2).

O crescimento das plantas de E. densa foi menor nos tratamentos que receberam inóculo de $F$. graminearum, tendo a biomassa fresca nestas plantas tido valores menores do que em plantas não inoculadas (Figura 1).

Vale ressaltar que apenas o arroz moído (não colonizado pelo fungo), também, interferiu na produção de biomassa reduzindo a mesma, em aproximadamente $1 / 3$, a partir da concentração de 0,9 g/l (Figura 1). O fato ocorre, provavelmente, devido ao aumento da Demanda Bioquímica de Oxigênio (DBO) nos referidos tratamentos. Espera-se que, quando aplicado em reservatórios, o efeito da DBO seja minimizado, devido à diluição e que o fungo continue atuando com eficiência. Análises de risco deverão ser realizadas antes da liberação deste agente de controle biológico em reservatórios, considerando a formulação em arroz-parboilizado.

Poucas são as referências encontradas na literatura, relacionadas à determinação da concentração de micélio para inoculação. Boyette et al. (1991), estudando a eficiência de controle de Datura stromonium L. com Alternaria crassa (Sacc.) Rands., obtiveram bons resultados com micélio fresco na proporção de $56 \mathrm{Kg} / \mathrm{ha}$ (aproximadamente $28 \mathrm{~g} / \mathrm{l}$ ). Apesar das formulações de inóculo a base de conídios serem mais eficientes (Charudattan, 1990), a produção de bioherbicidas a baixos custos é facilitada pela utilização de micélio fresco (Boyette et al., 1991) e ou massa micelial produzida em arroz (Nachtigal, 2000).

Nachtigal (2000) estudou o efeito de diferentes concentrações de inóculo de $F$. graminearum $(0,0$ g/l a 1,0 g/l variando de um décimo cada tratamento) e verificou um aumento considerável na severidade de doença na faixa de 0,1 a $0,5 \mathrm{~g} / \mathrm{l}$ tanto para $E$. densa como para $E$. najas e recomendou a concentração de $0,7 \mathrm{~g} / \mathrm{l}$ para ser utilizada como bioherbicida. No presente trabalho, corroborando com os dados obtidos por Nachtigal (2000), os tratamentos de 0,5 a 1,4 g/l apresentaram os melhores resultados. Contudo concentrações superiores a $0,9 \mathrm{~g} / \mathrm{l}$ podem interferir de forma negativa na qualidade da água, devido ao aumento na DBO, sendo o mais indicado a utilização de concentrações de 0,5 a $0,7 \mathrm{~g} / \mathrm{l}$.

TABELA 2 - Influência da concentração do inóculo na severidade de doença causada por Fusarium graminearum em plantas de Egeria densa

\begin{tabular}{|c|c|c|c|c|c|c|}
\hline \multirow{2}{*}{$\begin{array}{l}\text { Concentracao de } \\
\text { Inóculo (g/l) I }\end{array}$} & \multicolumn{4}{|c|}{ Área Foliar com Sintomas (\%) } & \multirow{2}{*}{$\mathbf{R}^{2}$} & \multirow{2}{*}{ Equações das Curvas de Progresso da Doença ${ }^{\text {III }}$} \\
\hline & 2 Dias $^{\mathrm{II}}$ & 4 Dias $^{I I}$ & 6 Dias $^{I I}$ & 8 Dias $^{\text {II }}$ & & \\
\hline Testemunha & 0,00 & 0,00 & 0,00 & 0,00 & - & - \\
\hline 0,1 & 0,00 & 0,00 & 0,00 & 0,00 & - & - \\
\hline 0,2 & 0,00 & 0,00 & 0,00 & 1,48 & - & - \\
\hline 0,3 & 0,00 & 0,00 & 2,22 & 2,22 & - & - \\
\hline 0,4 & 0,00 & 0,00 & 2,22 & 2,96 & - & - \\
\hline 0,5 & 0,00 & 1,48 & 4,44 & 8,15 & 0,989 & $\mathrm{Y}=\left[13,623 / 1+\mathrm{e}^{(\mathrm{X}-6,803) / 17,843}\right]+12,758$ \\
\hline 0,6 & 0,00 & 1,59 & 7,41 & 8,89 & 0,978 & $\mathrm{Y}=\left[13,751 / 1+\mathrm{e}^{(\mathrm{X}-5,864) / 0,085}\right]+8,89$ \\
\hline 0,7 & 1,48 & 4,44 & 14,07 & 24,44 & 0,967 & $\mathrm{Y}=\left[27,977 / 1+\mathrm{e}^{(\mathrm{X}-6,115) / 1,111}\right]+28,781$ \\
\hline 0,8 & 1,48 & 4,44 & 12,59 & 22,22 & 0,999 & $\mathrm{Y}=\left[27,607 / 1+\mathrm{e}^{(\mathrm{X}-6,332) / 1,289}\right]+28,161$ \\
\hline 0,9 & 2,22 & 2,96 & 19,26 & 31,11 & 0,999 & $\mathrm{Y}=\left[29.999 / 1+\mathrm{e}^{(\mathrm{X}-5,833) / 0,504}\right]+31,504$ \\
\hline 1,0 & 2,96 & 14,07 & 28,89 & 45,19 & 0,985 & $\mathrm{Y}=\left[99,260 / 1+\mathrm{e}^{(\mathrm{X}-6,904) / 3,016}\right]+85,899$ \\
\hline 1,1 & 2,96 & 11,11 & 25,19 & 36,30 & 0,987 & $\mathrm{Y}=\left[43,508 / 1+\mathrm{e}^{(\mathrm{X}-5,412) / 1,461}\right]+42,628$ \\
\hline 1,2 & 2,96 & 12,59 & 28,15 & 42,96 & 0,967 & $\mathrm{Y}=\left[60,333 / 1+\mathrm{e}^{(\mathrm{X}-5,823) / 1,801}\right]+56,839$ \\
\hline 1,3 & 5,93 & 13,33 & 33,33 & 48,89 & 0,969 & $\mathrm{Y}=\left[49,676 / 1+\mathrm{e}^{(\mathrm{X}-5,623) / 1,086}\right]+53,897$ \\
\hline 1,4 & 8,15 & 23,70 & 44,44 & 60,74 & 0,976 & $\mathrm{Y}=\left[78,219 / 1+\mathrm{e}^{(\mathrm{X}-5,092) / 1,839}\right]+74,092$ \\
\hline
\end{tabular}

${ }^{\mathrm{I}}$ Concentração em $\mathrm{g} / \mathrm{l}$ de micélio na densidade de $10 \mathrm{~g} / \mathrm{cm}^{3}$.

II Média dos Índices de Infecção calculados segundo Mckinney (1923).

III Dados submetidos à análise não linear, utilizando-se a equação de Boltzmann. As equações das curvas de progresso da doença foram obtidas utilizando-se o programa Origin ${ }^{\circledR} 6.0$. 


\section{Idade da Planta}

Ambas as espécies inoculadas, em todas as idades avaliadas, não apresentaram sintomas da doença no segundo dia após a inoculação (Figura 2 - I a IV). No quarto dia após a inoculação sintomas fracos da doença, sendo que a $E$. najas mostrou-se mais sensível ao fungo (Figura 2 - III e IV). As testemunhas não inoculadas de cada idade não foram afetadas pelo arroz moído adicionado à solução nutritiva.

Ponteiros de plantas de ambas as espécies (Figura 2 - I) apresentaram maior severidade de sintomas no sexto e oitavo dias após a inoculação. Os segmentos correspondentes às Idades 2 e 3 (Figura 2 - II e III) apresentaram severidade de sintomas menor que os ponteiros (Figura 2 - I), porém maior, que a Idade 4 (Figura 2 - IV).

As testemunhas apresentaram maior ganho de biomassa fresca que os respectivos tratamentos inoculados, em média $27 \%$ para E. densa e $30 \%$ para E. najas, contudo, os segmentos correspondentes à Idade 4 apresentaram menor ganho (média de $7 \%$ para $E$. densa e $17 \%$ para $E$. najas) do que para as demais idades.

Segmentos de plantas de ambas as espécies das Idades 2 e 3 (Figura 2 - II e III) mostraram-se mais sensíveis que os ponteiros (Figura 2 - I) e menos sensíveis que segmentos da Idade 4 (Figura 2 -IV), diferindo estatisticamente com relação ao ganho de biomassa fresca dos mesmos, aos oito dias após a inoculação. Sendo que ponteiros de E. najas. não apresentaram crescimento (Figura 2 - I), sendo totalmente destruídos pelo fungo.

A idade da planta daninha alvo, na qual ela se torna mais susceptível, deve ser definida para cada associação patógeno/hospedeiro. No controle biológico de plantas terrestres, Mintz et al. (1992), estudando o controle de Amaranthus albus L. com Aposphaeria amaranthi (Peck) J.M. Hook, verificaram que plantas no estádio de quatro folhas definitivas foram mais susceptíveis à doença. Neste caso, plantas muito jovens não apresentaram níveis satisfatórios de doença, sugerindo resistência de fase juvenil.

Em contrapartida, Anoda cristata (L.) Schltdl. e Senna obtusifolia (L.) H.S. Irwin \& Barneby, que têm sido alvo de

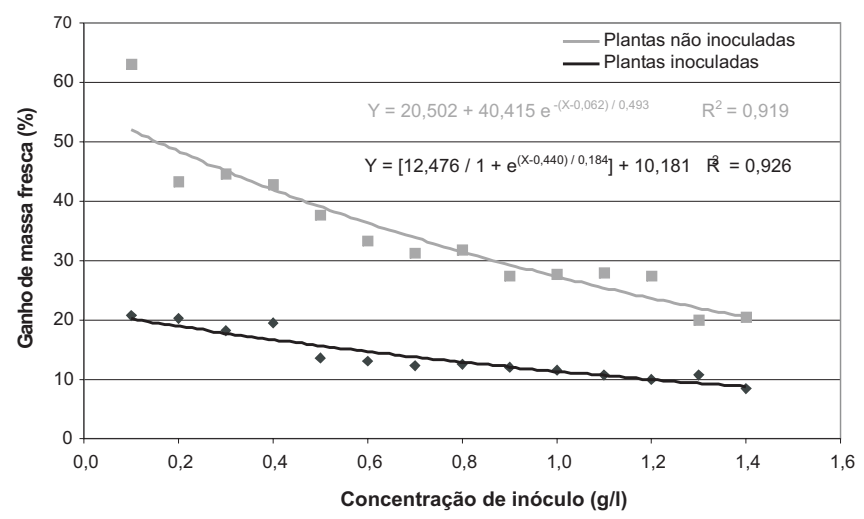

FIG. 1 - Influência da concentração do inóculo na produção de biomassa fresca em plantas de Egeria densa inoculadas com Fusarium graminearum comparadas com plantas não inoculadas.
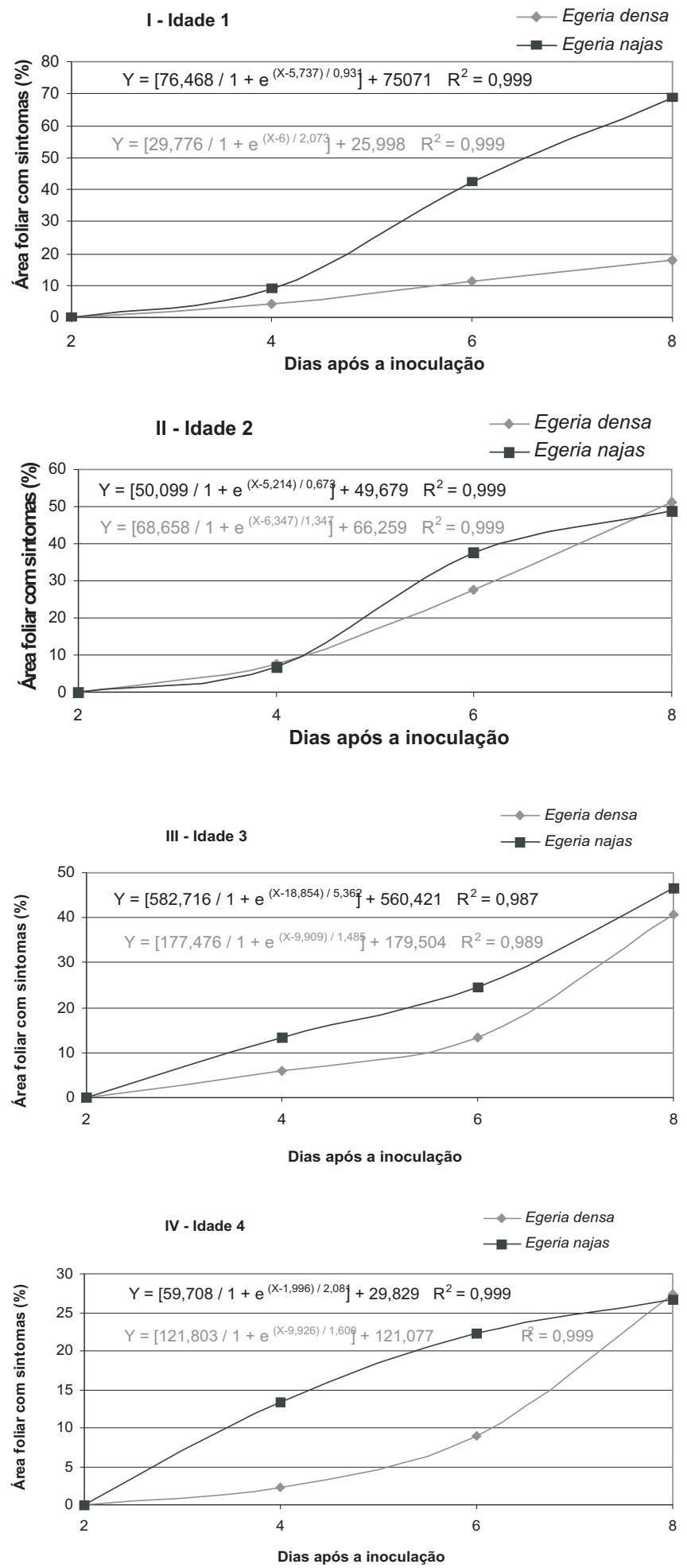

FIG. 2 - Influência da idade de plantas de Egeria densa e E. najas na infetividade de Fusarium graminearum. Plantas, com no mínimo 35 $\mathrm{cm}$ de comprimento, foram excisadas em diversos segmentos, com 8 $\mathrm{cm}$ de comprimento, correspondentes às idades de crescimento. Para efeitos de avaliação foi considerada: Idade 1 - ponteiros de $8 \mathrm{~cm}$ de ambas as espécies, que corresponderiam aos tecidos mais jovens; Idade 2 - segmentos coletados de $8 \mathrm{~cm}$ a $16 \mathrm{~cm}$ de profundidade; Idade 3 - segmentos coletados de $16 \mathrm{~cm}$ a $24 \mathrm{~cm}$ de profundidade; e idade 4 - segmentos coletados de $24 \mathrm{~cm}$ a $32 \mathrm{~cm}$ de profundidade. 
Influência da concentração de inóculo e da idade da planta...

estudos para o controle biológico, através de inoculações inundativas com Alternaria macrospora Zimm. (Walker, 1981) e A. cassiae Jurair \& A. Khan (Figueiredo et al., 1992a; Figueiredo et al., 1992b), respectivamente, são exemplos clássicos de plantas daninhas consistentemente controladas na fase inicial de desenvolvimento.

No patossistema tiririca (Cyperus rotundus L.) $\mathrm{x}$ Cercospora caricis Oudem., plantas mais velhas com quatro semanas de idade foram mais afetadas pela doença tendo apresentado $41 \%$ de folhas mortas e $66 \%$ de folhas infetadas (Borges Neto, 1997; Borges Neto et al., 1997; Borges Neto al., 2000).

A melhor época de inoculação para o desenvolvimento de níveis adequados de doença é bastante variável, podendo envolver, além da resistência da hospedeira, vários outros fatores, tais como temperatura, umidade, diluição e pressão de inóculo, interagindo entre si.

Dentro da linha de pesquisa de controle biológico de plantas aquáticas submersas, praticamente inexistem referências de experimentos que relacionam severidade de doença com idade da planta, provavelmente, devido à dificuldade de determinar a real idade das plantas-teste, sendo possível, apenas, comparar vários segmentos de um conjunto de plantas, como foi realizado no presente trabalho.

Segundo Nachtigal (2000), F. graminearum reside inconspicuamente dentro dos tecidos de E. densa e E. najas. Este fato é corroborado pela irregularidade de recuperação do patógeno a partir de procedimentos rotineiros de isolamento. O isolado FCAV\#940, utilizado no presente estudo, parece não ter habilidade de penetrar nos tecidos das plantas aquáticas submersas, a despeito de suas folhas apresentarem cutícula e epiderme finas que, tecnicamente, não constituiriam barreira mecânica ao ingresso do patógeno (Nachtigal, 2000). Ainda conforme Nachtigal (2000), as plantas aquáticas submersas não possuem estômatos ou os mesmos não são funcionais, conseqüentemente $F$. graminearum pode não ter outra porta de entrada a não ser por meio de ferimentos (Nachtigal, 2000). Este fato pode explicar, em parte, a maior suscetibilidade dos segmentos correspondentes às Idades 2 e 3, excisadas no ápice e base. Por outro lado, os segmentos correspondentes a Idade 4, apesar de possuírem, também, duas portas de entrada para o patógeno, não apresentaram sintomas tão severos quanto aos demais tratamentos, mostrando que fatores relacionados à idade da planta influenciam diretamente na patogênese.

As informações aqui obtidas poderão auxiliar na interpretação de dados relacionados ao modo de ação do fungo e na identificação das variáveis que, uma vez alteradas, poderão contribuir para maior eficiência deste organismo como agente de biocontrole.

\section{REFERÊNCIAS BIBLIOGRÁFICAS}

ANDERSON, L.W.J. Can Egeria densa be eradicated? Yes, but is it worth it? Abstracts. Annual Meeting of Aquatic Plant Managemente Society, 38, 1998, Memphis, 1998. p.18.
BORGES NETO, C.R. Estudos sobre Cercospora caricis Oudem. como agente potencial de biocontrole da tiririca (Cyperus rotundus L.). 1997. Tese (Mestrado em Fitopatologia). Universidade de Brasília, Brasília, 1997.

BORGES NETO, C.R., MELLO, S.C.M. \& RIBEIRO, Z.M.A. Estudo das interações Cercospora caricis x tiririca (Cyperus rotundus), sob condições de casa de vegetação. Fitopatologia Brasileira 22:252. 1997. (Resumo)

BORGES NETO, C.R., MELLO, S.C.M. \& RIBEIRO, Z.M.A., ÁVILA, Z.R., MALTY, J.S. \& FONTES, E.M.G. Influência da idade da planta, período de umidificação e concentração de inóculo no desenvolvimento de sintomas provocados por Cercospora caricis em tiririca. Fitopatologia Brasileira 25:138-142. 2000.

BOYETTE, C.D., WEIDEMANN, G.J., TE BEEST, D.O. \& QUIMBY JR., P.C. Biological control of jimsonweed (Datura stramonium) with Alternaria crassa. Weed Science 39:678-681. 1991.

CLARK, R.B. Characterization of phosphate of intact maize roots. Journal of Agricultural and Food Chemistry 23:458-460. 1975.

CORRELL, D.S. \& CORRELL, H.B. Aquatic and wetland plants of southwestern United States. Stanford:Stanford University Press, 1975.

CHARUDATTAN, R. \& WALKER, H.L. Biological control of weeds with plant pathogens. New York: John Wiley, 1982.

CHARUDATTAN, R. Assessment of efficacy of mycoherbicide candidates. Proceedings. VII International Symposium on Biological Control of Weeds, 1990, Rome. Istituto Sperimentale per la Patologia Vegetale, 1990. pp.455-64.

FIGUEIREDO, G., FONTES, E.M.G., TEIXEIRA, C.A.D. \& PAIS, J.S.O. Levantamento e seleção de patógenos para o controle biológico de fedegoso (Senna obtusifolia, Leguminosae). Fitopatologia Brasileira 17:169. 1992a. (Resumo)

FIGUEIREDO, G., FONTES. E.M.G. \& CHARUDATTAN, R. Ocorrência de Alternaria cassiae e seu potencial como agente de controle biológico do fedegoso, Senna (Cassia) obtusifolia no Brasil. III Simpósio de Controle Biológico, 1992, Águas de Lindóia. Resumos. EMBRAPA-CNPDA. 1992b. p.183.

LORENZI, H. Plantas daninhas do Brasil: Terrestres, aquáticas, parasitas e tóxicas. 3nd ed. Nova Odessa - SP: Instituto Plantarum de Estudos da Flora Ltda., 2000.

MCKINNEY, H.H. Influence of soil temperature and moisture on infection of wheat seedlings by Helminthosporium sativum. Journal of Agricultural Research 26:194-218. 1923.

MENDES, D. Efeitos de herbicidas sobre alguns aspectos biológicos de Fusarium graminearum (isolado FCAV\#940), agente potencial de controle biológico de Egeria densa e Egeria najas. (Dissertação de Mestrado). Jaboticabal, FCAV/UNESP, 2002.

MINTZ, A.S., HEINY, D.K. \& WEIDEMANN, G.J. Factors influencing the biocontrol of tumble pigweed (Amaramthus albus) with Aposphaeria amaranthi. Plant Disease 76:267-269. 1992.

NACHTIGAL, G.F. Desenvolvimento de agente de controle biológico microbiano de Egeria densa e Egeria najas. 2000. 160f. Tese de Doutorado em Produção Vegetal) - Faculdade de Ciências Agrárias e Veterinárias, Universidade Estadual Paulista, Jaboticabal, 2000.

PITELLI, R.A. Macrófitas aquáticas no Brasil, na condição de problemáticas. Resumos. Workshop Controle de Plantas Aquáticas, 1998, Brasília. IBAMA, 1998. pp.12-15.

PITELLI, R.A., PITELLI, R.L.C.M. \& MARCONDES, D.A.S. 


\section{C.R. Borges Neto et al.}

Controle biológico de macrófitas aquáticas. Resumos. Workshop Ecologia e Manejo de Macrófitas Aquáticas, Maringá. Sociedade Brasileira de Limnologia, UEM, Nupelia, PEA, Eletrobrás, 2000. p.23.

TANAKA, R.H. Prejuízos provocados pelas plantas aquáticas. Resumos. Workshop sobre Controle de Plantas Aquáticas, Brasília. 1998, pp.36-38.

TE BEEST, D.O. Microbial control of weeds. Chapman and Hall, NY. 1991.
TE BEEST, D.O. \& TEMPLETON, G.E. Mycoherbicides: Progress in the biological control of weeds. Plant Disease 69:6-10. 1985.

VAN DEN BOSH, R., MESSENGER, P.S. \& GUTIERREZ, A. An introduction to biological control. New York:Plenum Press, 1987.

WALKER, H.L. Factors affecting biological control of spurred anoda (Anoda cristata) with Alternaria macrospora. Weed Science 29:505507. 1981. 